\title{
Fluid structure interactions of a pitching wing at high angle of attack
}

\author{
G. Acher ${ }^{1 *}$, Patrick Braud ${ }^{1 *}$, Ludovic Chatellier ${ }^{1 *}$, Lionel Thomas ${ }^{1 *}$, Laurent \\ David $^{1 *}$ \\ ${ }^{1}$ Institut PPrime, UPR 3346, CNRS-Universit de Poitiers-ENSMA, France \\ *laurent.david@univ-poitiers.fr
}

\begin{abstract}
This paper deals with the study of the flow around a pitching wing at high angle of attack. Different pitching amplitudes and frequencies are studied using DIC and LPT measurements. The Fluid Structure Interactions are shown and exhibit that the vortex shedding could be reduced by actuating the wing at specific frequencies.
\end{abstract}

\section{Introduction}

In high incidence conditions, wings are subjected to large vortex detachments which promote the dynamic stall leading to a sudden drop in lift. Under such conditions it is often necessary to increase the lifting surface so that the effective angle of attack is reduced, or prevent vortex shedding by using actuators such as plasma actuators or micro jets with the objective of moving the point of detachment away from the leading edge. Another way to reduce these massive detachments is to force pitching of the profile with a low amplitude around the characteristic frequencies of this vortex shedding. The object of the study is therefore to study the modifications of the flow in the case of a wing pitching around a position of high incidence and to measure the effects of this oscillation on the near wake, and simultaneously to look at the mechanical effects produced on the wing. Measurements of the 3D deformation of a NACA0015 flexible wing profile installed at a large angle of attack and pitching with small amplitude around the natural vortex shedding frequency at a Reynold number of $10^{5}$ are applied simultaneously with 3D flow measurements. Wing time-deformation, volumetric instantaneous velocity fields are measured and correlated to show mutual influence between the flow and the wing.

\section{Results}

A wing profile of $146 \mathrm{~mm}$ span is placed at 15 angle of attack in the test section of a hydrodynamic tunnel. The NACA0015-type wing profile, based on a $80 \mathrm{~mm}$ chord, is built of a $140 \mathrm{~mm}$ long straight section and its tip is formed by the rotation of the profile around the chord, adding $6 \mathrm{~mm}$ of wingspan, at the position of maximum thickness. The profile is oscillating with an amplitude of 2 and 4 around the initial angle of incidence at different frequencies around the natural frequency of the vortex shedding $(\mathrm{f}=5.82 / 8.93 / 12.50$ $\mathrm{Hz}$ ). The surface of the wing is monitored by two high speed cameras so that both the pitching motion and deformations are characterized. Four other high speed cameras are used to investigate the instanteous flow fields using Lagrangian Particle Tracking (Figure 1).

Time resolved estimation of the wing motion and deformation is obtained using the PIV/DIC method described by Chatellier et al. (2013),. The technique is based on maximizing the correspondence of two back-projected images on the estimated solid surface. In order to follow the deformation of the wing profile in the test section, a speckle pattern is painted on its pressure side so that the two high speed cameras placed under the water tunnel image the full wingspan. An example of results is presented in the Figure 2.

The flow velocity field is measured using Shake-The-Box algorithm Schanz et al. (2016), in order to provide 3D time-resolved particle tracks from the four camera recordings. Velocity and acceleration are extracted (Figure 3) and allow to calculate the main characteristics of the flow in terms of vorticity, Q criteria 


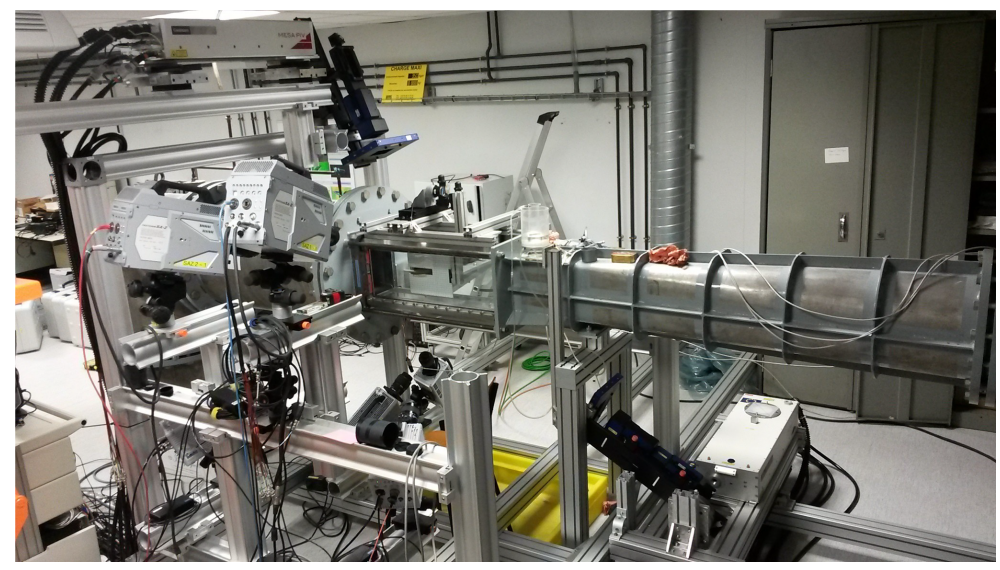

Figure 1: Tomographic PIV and stereo-DIC setup as used for the pitching wings experiments

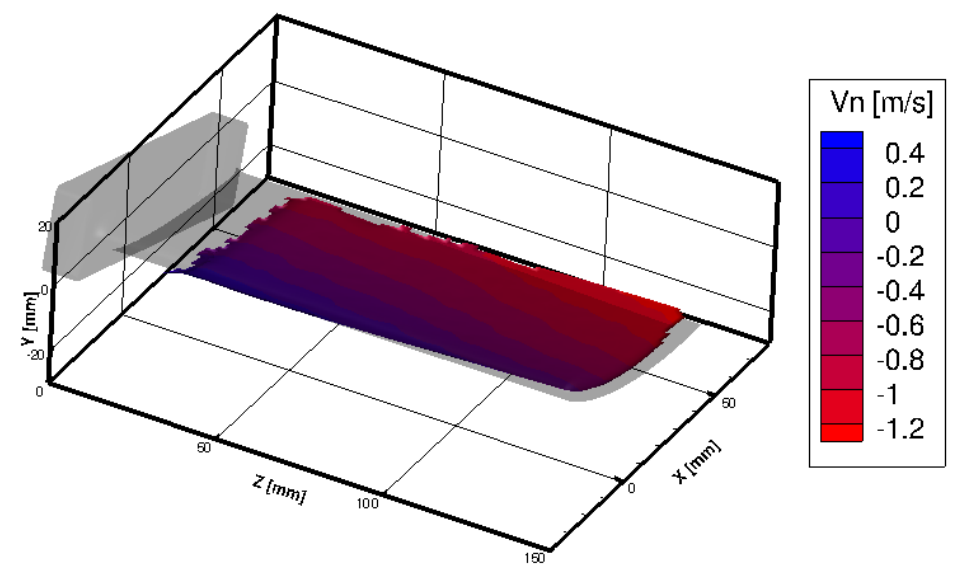

Figure 2: Three-dimensional view of the reconstructed lower surface of the wing superimposed with the undeformed wing shape

and pressure-from-PIV estimates. Correlation between the solid and fluid databases are then calculated to estimate the interaction between the fluid and structure and allow to understand the effect of the flexible wing for wake minimization and drag reduction.

\section{Conclusions}

The study of the flow around a pitching wing at high angle of attack has been carried out for different amplitudes and frequencies of the wing. DIC and LPT measurements have been recorded to understand the Fluid Structure Interactions and exhibit that the vortex shedding is modified with specific frequencies and allow the drag reduction.

\section{Acknowledgements}

The authors would like to thank the project HOMER : Holistic Optical Metrology for Aero-Elastic Research under the European Unions Horizon 2020 research and innovation programme (grant agreement No 648161)and the CPER NUMERICS program for their fundings. 


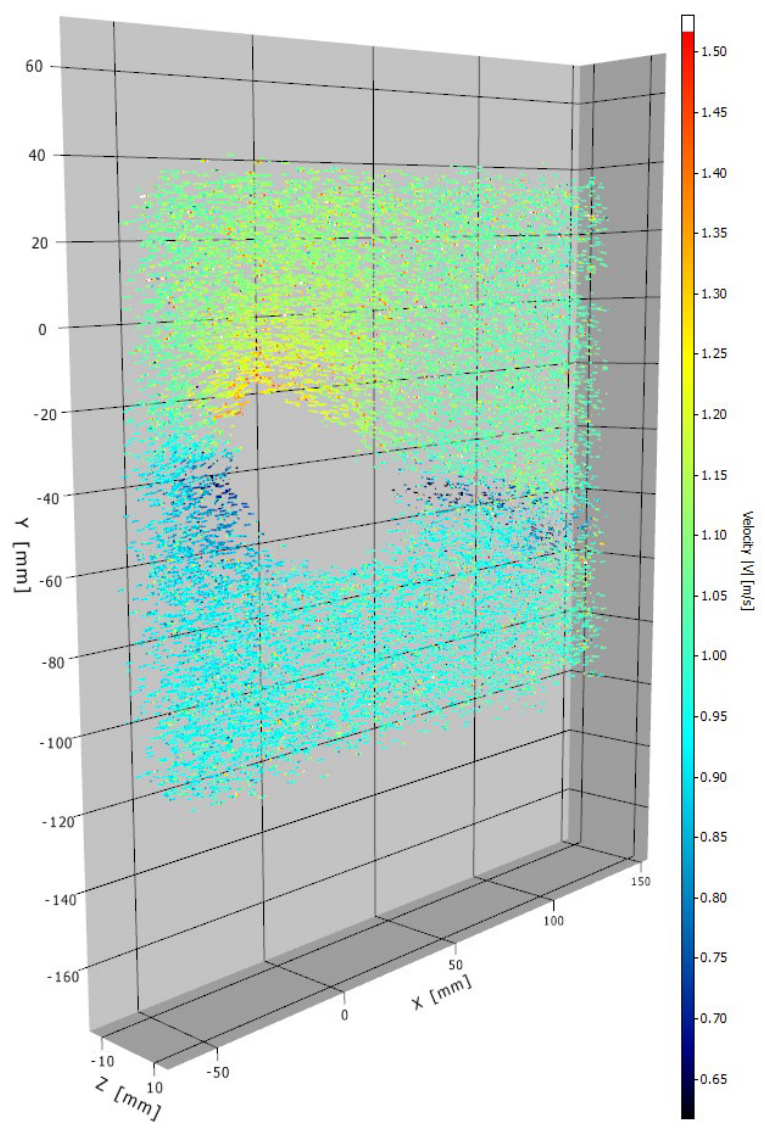

Figure 3: LPT sample measurements on the pitching flexible wing $(\mathrm{z}=70 \mathrm{~mm}, \mathrm{f}=5.89 \mathrm{~Hz},=2)$

\section{References}

Chatellier L, Jarny S, Gibouin F, and David L (2013) A parametric piv/dic method for the measurement of free surface flowsa parametric piv/dic method for the measurement of free surface flows. Experiments in Fluids 54:1488

Schanz D, Gesemann S, and Schrder A (2016) Shake-the-box: Lagrangian particle tracking at high particle image densities. Experiments in fluids 57:1-27 